DOE/ER/40561-179-INT94-12-04

\title{
Magnetization of Ferromagnetic Clusters
}

\author{
Naoki Onishi \\ Institute of Physics, College of Arts and Sciences, \\ University of Tokyo, Komaba, Meguro-ku, Tokyo, 153 Japan \\ George Bertsch \\ Department of Physics and Institute for Nuclear Theory, FM-15 \\ University of Washington, Seattle, WA 98195 \\ and \\ Kazuhiro Yabana \\ Department of Physics, Faculty of Science, Niigata University \\ Igarashi, Niigata-city, Niigata, 950-21 Japan
}

\section{PREPARED FOR THE U.S. DEPARTMENT OF ENERGY UNDER GRANT DE-FG06-90ER40561}

This report was prepared as an account of work sponsored by the United States Government. Neither the United States nor any agency thereof, nor any of their employees, makes any warranty, express or implied, or assumes any legal liability or responsibility for the accuracy, completeness, or usefulness of any information, apparatus, product, or process disclosed, or represents that its use would not infringe privately owned rights. Reference herein to any specific commercial product, process, or service by trade name, mark, manufacturer, or otherwise, does not necessarily constitute or imply its endorsement, recommendation, or favoring by the United States Government or any agency thereof. The views and opinions of authors expressed herein do not necessarily state or reflect those of the United States Government or any agency thereof.

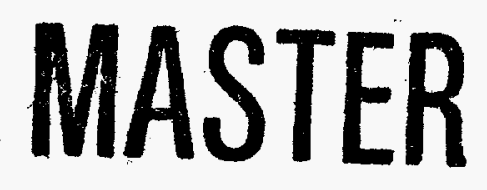




\section{DISCLAIMER}

Portions of this document may be illegible in electronic image products. Images are produced from the best available original document. 


\title{
Magnetization of Ferromagnetic Clusters
}

\author{
Naoki Onishi \\ Institute of Physics, College of Arts and Sciences, \\ University of Tokyo, Komaba, Meguro-ku, Tokyo, 153 Japan \\ George Bertsch \\ Department of Physics and Institute for Nuclear Theory, FM-15 \\ University of Washington, Seattle, WA. 98195 \\ and \\ Kazuhiro Yabana \\ Department of Physics, Faculty of Science, Niigata University \\ Igarashi, Niigata-city, Niigata, 950-21 Japan
}

\begin{abstract}
The magnetization and deflection profiles of magnetic clusters in a Stern-Gerlach magnet are calculated for conditions under which the magnetic moment is fixed in the the intrinsic frame of the cluster, and the clusters enter the magnetic field adiabatically. The predicted magnetization is monotonic in the Langevin parameter, the ratio of magnetic energy $\mu_{0} B$ to thermal energy $k_{B} T$. In low field the average magnetization is $2 / 3$ of the Langevin function. The high-field moment approaches saturation asymptotically as $B^{-1 / 2}$ instead of the $B^{-1}$ dependence in the Langevin function.
\end{abstract}

\section{Introduction}

The magnetization of small clusters composed of ferromagnetic elements have been studied by their deflection in a Stern-Gerlach magnet [1-5]. It is reasonable to suppose that the magnetic field changes slowly, so that the clusters respond adiabatically to the changing fields going in and out of the magnet. If the ferromagnetic element has a high coercivity and the clusters are cold, the magnetic moment will be frozen in the cluster during the traversal of the Stern-Gerlach apparatus. We consider here this limiting case.

In a previous paper [7], two of us studied this problem using a quantum mechanical description of the rotor. However, the typical angular momenta are high enough so that quantization of angular momentum does not play a significant role. In this paper, we treat the problem completely classically. The same classical Hamiltonian was considered by the authors of ref. [3] who numerically integrated the equations of motion to find the adiabatic magnetization. Another numerical study of the frozen adiabatic limit was presented in [8]. Our approach is more analytic; we shall make use of an adiabatic invariant in the Hamiltonian of an axially symmetric rotor. The existence of the invariant allows the problem to be solved in terms of the elliptic integrals of the first and second kind. Because of the reduction to integrals, we are also able to extract the limiting behavior in low and high magnetic fields. Our results agree with the quantum study [7] but are at variance with the conclusions 
of ref. [8].

\section{Rigid body motion in a magnetic field}

We first write down the general Hamiltonian for a magnetized rigid body. In terms of the principal moments of inertia $\mathcal{J}_{1}, \mathcal{J}_{2}$ and $\mathcal{J}_{3}$, the magnetic moment $\mu_{0}$ and the field strength $B$, the Lagrangian is given by

$$
\mathcal{L}=\frac{1}{2} \sum_{k=1}^{3} \mathcal{J}_{k} \omega_{k}^{2}+\mu_{0} B \cos \Theta(\theta, \psi)
$$

Here $\Theta(\theta, \phi)$ is the angle between the directions of the magnetic moment and the field,

$$
\cos \Theta=\cos \theta_{0} \cos \theta-\sin \theta_{0} \sin \theta \cos \left(\psi+\psi_{0}\right) .
$$

where $\left(\theta_{0}, \psi_{0}\right)$ is the orientation of the spin in the body-fixed frame. The angular velocity $\omega_{k}$ along the $k$-axis of the body-fixed frame is expressed as a linear combination of the time derivatives of Euler's angles,

$$
\omega_{k}=\sum_{\mu} a_{k \mu}(\theta \psi) \dot{\theta}_{\mu} \quad \text { with } \quad(\theta, \phi, \psi)=\left(\theta_{1}, \theta_{2}, \theta_{3}\right)
$$

This Lagrangian has two constants of motion. One is the energy $E$, given by

$$
E=\frac{1}{2} \sum_{k=1}^{3} \mathcal{J}_{k} \omega_{k}^{2}-\mu_{0} B \cos \Theta(\theta, \psi)
$$

The Euler angle $\phi$ is a cyclic coordinate, and this gives rise the second constant of motion, the angular momentum component along the magnetic field direction. Hence

$$
\frac{\partial \mathcal{L}}{\partial \dot{\phi}}=-\sin \theta \cos \psi m_{1}+\sin \theta \sin \psi m_{2}+\cos \theta m_{3}=m_{z}
$$

is an integral of motion. The dynamical variables $m_{k}$ 's defined as

$$
m_{k}=\mathcal{J}_{k} \omega_{k},
$$

are components of angular momentum along the principal axis of the moment of inertia. The modulus of the angular momentum, $I=\sqrt{m_{1}^{2}+m_{2}^{2}+m_{3}^{2}}$, is no longer constant of motion owing to the anisotropic term $\mu_{0} B \cos \Theta$.

For a general set of parameters $\left(\mathcal{J}_{1} \mathcal{J}_{2} \mathcal{J}_{3} \theta_{0} \psi_{0}\right)$, the motion is not integrable and can only be solved numerically. However, the simpler case in which the rotor has a symmetry axis does have an additional constant of motion and therefore can be solved completely. Taking two of the moments of inertia equal, $\mathcal{J}_{1}=\mathcal{J}_{2}$, and assuming that the body is magnetized along the symmetry axis $\left(\theta_{0}=0\right)$, the Langrangian becomes

$$
\mathcal{L}_{\text {sym }}=\frac{\mathcal{J}_{1}}{2}\left(\dot{\theta}^{2}+\sin ^{2} \theta \dot{\phi}^{2}\right)+\frac{\mathcal{J}_{3}}{2}(\dot{\psi}+\cos \theta \dot{\phi})^{2}+\mu_{0} B \cos \theta .
$$


Since this Lagrangian does not include the angle $\psi$, it has the additional constant of motion,

$$
\frac{\partial \mathcal{L}_{\mathrm{sym}}}{\partial \dot{\psi}}=\mathcal{J}_{3}(\dot{\psi}+\cos \theta \dot{\phi})=m_{3} .
$$

For the symmetric rotor, eq. (2.5) becomes

$$
\mathcal{J}_{1} \sin ^{2} \theta \dot{\phi}+m_{3} \cos \theta=m_{z} .
$$

Eq. (2.8) and (2.9) may then be solved for $\dot{\phi}$ and $\dot{\psi}$ to obtain

$$
\dot{\phi}=\frac{m_{z}-m_{3} \cos \theta}{\mathcal{J}_{1} \sin ^{2} \theta},
$$

and

$$
\dot{\psi}=\frac{m_{3}}{\mathcal{J}_{3}}-\cos \theta \frac{m_{z}-m_{3} \cos \theta}{\mathcal{J}_{1} \sin ^{2} \theta} .
$$

Insertion of these solutions into the energy function yields

$$
\frac{\mathcal{J}_{1}}{2} \dot{\theta}^{2}+U_{\text {eff }}(\theta)=E^{\prime},
$$

where the effective potential is given by

$$
U_{\text {eff }}(\theta)=\frac{\left(m_{z}-m_{3} \cos \theta\right)^{2}}{2 \mathcal{J}_{1} \sin ^{2} \theta}-\mu_{0} B \cos \theta,
$$

and the shifted energy $E^{\prime}$ is defined as

$$
E^{\prime}=E-\frac{m_{3}^{2}}{2 \mathcal{J}_{3}}
$$

Through the two constants of motion, the dynamical variable $\theta$ is separated from two others, and the motion of the present system turns out to be completely integrable. The time dependence of a back and forth motion of $\theta(t)$ is expressed in an integral form

$$
\int \mathrm{d} t=\mathcal{J}_{1} \int \frac{\mathrm{d} \theta}{\sqrt{2 \mathcal{J}_{1}\left\{E^{\prime}-U_{\text {eff }}(\theta)\right\}}} .
$$

By a variable transformation to $u=\cos \theta$, the integral becomes

$$
t-t_{0}=\mathcal{J}_{1} \int \frac{\mathrm{d} u}{\sqrt{f(u)}},
$$

where the function $f(u)$ is a cubic polynomial in $u$,

$$
f(u)=(e+h u)\left(1-u^{2}\right)-\left(m_{\mathbf{z}}-m_{3} u\right)^{2},
$$

with constants

$$
e=2 \mathcal{J}_{1} E^{\prime} \quad \text { and } \quad h=2 \mathcal{J}_{1} \mu_{0} B
$$


The polynomial $f$ has three roots $u_{i}$,

$$
f(u)=h\left(u_{0}-u\right)\left(u_{1}-u\right)\left(u_{2}-u\right)=0 .
$$

The periodic motion called nutation takes place in the interval between two roots $\left[u_{1}, u_{2}\right]$ that lie in the range $[-1,1]$. The period of the nutation is given by the integral

$$
\tau=2 \mathcal{J} \int_{u_{1}}^{u_{2}} \frac{\mathrm{d} u}{\sqrt{f(u)}} .
$$

The effective magnetization of the rotor is $\mu=\mu_{0} \bar{u}$ where the polarization $\bar{u}$ is calculated as the time average

$$
\bar{u}=\frac{1}{\tau} \int_{0}^{\tau} \cos \theta \mathrm{d} t=\int_{u_{1}}^{u_{2}} \frac{u \mathrm{~d} u}{\sqrt{f(u)}} / \int_{u_{1}}^{u_{2}} \frac{\mathrm{d} u}{\sqrt{f(u)}} .
$$

These integrals may be expressed in terms of the complete elliptic integrals of the first and second kind, $K(\nu)$ and $E(\nu)$, respectively. The period of the nutation and the average polarization are given by

$$
\begin{gathered}
\tau=\frac{2}{\sqrt{h\left(u_{2}-u_{0}\right)}} K(\nu), \\
\bar{u}=\frac{u_{2} K(\nu)+\left(u_{2}-u_{0}\right) E(\nu)}{K(\nu)},
\end{gathered}
$$

where $\nu=\left(u_{2}-u_{1}\right) /\left(u_{2}-u_{0}\right)$.

\section{Adiabatic invariance}

In the previous section, the time-averaged magnetization was obtained as a function of the variables $E, m_{3}$, and $m_{z}$. The latter two are constants of the motion and therefore can be specified by the initial conditions, but we need an additional condition to relate the energy in the field to the initial distribution. The quantity that is conserved when the particle enters the magnetic field adiabatically is the adiabatic invariant associated with the motion in the $\theta$ coordinate. This is

$$
J_{\theta}=2 \int_{\theta_{1}}^{\theta_{2}} P_{\theta} \mathrm{d} \theta=2 \int_{u_{1}}^{u_{2}} \frac{\sqrt{f(u)} \mathrm{d} u}{1-u^{2}},
$$

where $\theta_{1}, \theta_{2}$ or $u_{1}, u_{2}$ are the limits of motion for a given set of $\left(e, m_{z}, m_{3}, h\right)$, and $P_{\theta}$ is the momentum conjugate to the Euler angle $\theta$,

$$
P_{\theta}=\frac{\partial \mathcal{L}_{\text {sym }}}{\partial \dot{\theta}}=\mathcal{J}_{1} \dot{\theta}
$$


The magnetization defined by $\mu_{0} \bar{u}$ may also be calculated from the derivative of the energy with respect to magnetic field at fixed $J_{\theta}$,

$$
\left.\frac{\mathrm{d} e}{\mathrm{~d} h}\right)_{J_{\theta}}=\frac{\partial J_{\theta}}{\partial e} / \frac{\partial J_{\theta}}{\partial h}=-\bar{u} .
$$

For zero external magnetic field, $h=0$, the integral in eq.(3.24) may be evaluated analytically to give

$$
J_{\theta}\left(\varepsilon\left(I, m_{3}\right), m_{z}, m_{3}, 0\right)=2 \pi\left(I-\frac{\left|m_{\mathrm{z}}-m_{3}\right|}{2}-\frac{\left|m_{\mathrm{z}}+m_{3}\right|}{2}\right) .
$$

where $I$ is the total angular momentum defined earlier. Let us connect this with quantum mechanics, expressing $I, M_{3}, m_{z}$ and $J_{\theta}$ in units of $\hbar$. The above formula gives the number $n_{\theta}=J_{\theta} / 2 \pi$ as the minimum of the four numbers $\left(I \pm m_{z}, I \pm m_{3}\right)$. The $n_{\theta}$ corresponds to the number of nodes in the Wigner $d$-function $d_{m_{z} m_{3}}^{I}(\theta)$. Recall that the $d$-function is also the wave function of an axially symmetric rotor.

The adiabatic invariant $J_{\theta}$ relates the energy $E$ of the system in the magnetic field $B$ to the total angular momentum $I$ in zero field. This quantity was used in generalization of Bohr's quantization rule by Einstein and Ehrenfest. It is the variable associated with classical quantization of nutation.

In calculating ensemble averages, we will need to evaluate phase space integrals of the form

$$
\int d \Omega=\frac{1}{(2 \pi)^{3}} \int_{-\infty}^{\infty} \mathrm{d} P_{\theta} \int_{0}^{\pi} \mathrm{d} \theta \int_{-\infty}^{\infty} \mathrm{d} m_{z} \int_{0}^{2 \pi} \mathrm{d} \phi \int_{-\infty}^{\infty} \mathrm{d} m_{3} \int_{0}^{2 \pi} \mathrm{d} \psi
$$

This integral may also be expressed

$$
\frac{1}{\pi} \int_{0}^{\infty} \mathrm{d} I \int_{-I}^{I} \mathrm{~d} m_{z} \int_{-I}^{I} \mathrm{~d} m_{3} \int_{u_{1}}^{u_{2}} \frac{\mathrm{d} u}{\sqrt{\left(u_{2}-u\right)\left(u-u_{1}\right)}} .
$$

In deriving this we used the relation between $I$ and $P_{\theta}$,

$$
I^{2}=P_{\theta}^{2}+\frac{\left(m_{z}-m_{3} \cos \theta\right)^{2}}{\sin ^{2} \theta}+m_{3}^{2}
$$

The endpoints of integration in eq. (3.29) are given by

$$
u_{1,2}=\frac{m_{3} m_{\mathrm{z}} \pm \sqrt{\left(I-m_{3}\right)\left(I+m_{3}\right)\left(I-m_{\mathrm{z}}\right)\left(I+m_{\mathrm{z}}\right)}}{I^{2}} .
$$

Note the correspondance with quantum mechanics: the first three integrals in eq. (3.29) correspond to the familiar quantum mechanical sum over rotational states,

$$
\sum_{I=0}^{\infty} \sum_{m_{z}=-I}^{I} \sum_{m_{z}=-I}^{I}
$$

We can now construct a thermal ensemble labeling the states of motion by $m_{z}, m_{3}$, and $I$. Note that $I$ is only the angular momentum in zero field; at finite $B$ it is a label for the state having $J_{\theta}$ given by eq. (3.27). The Boltzmann 
factor in zero field is given by $\exp \left(-\varepsilon\left(I, m_{3}\right) / k_{B} T\right)$ where the zero field energy is $\varepsilon\left(I, m_{3}\right)=\left(I^{2}-m_{3}^{2}\right) / 2 \mathcal{J}_{1}+m_{3}^{2} / 2 \mathcal{J}_{3}$. The partition function for the rotor ensemble is

$$
Z(\beta)=\int_{0}^{\infty} \mathrm{d} I \int_{-I}^{I} \mathrm{~d} m_{z} \int_{-I}^{I} \mathrm{~d} m_{3} \mathrm{e}^{-\beta \varepsilon\left(I, m_{3}\right)}=2 \mathcal{J}_{1} \sqrt{2 \pi \mathcal{J}_{3}}\left(k_{\mathrm{B}} T\right)^{\frac{3}{2}}
$$

We may finally evaluate the average of an operator $\mathcal{O}$ in the adiabatic rotor ensemble as

$$
\langle\mathcal{O}\rangle_{\mathrm{ar}}=\frac{1}{Z(\beta)} \int_{0}^{\infty} \mathrm{d} I \int_{-I}^{I} \mathrm{~d} m_{z} \int_{-I}^{I} \mathrm{~d} m_{3} \mathcal{O} \mathrm{e}^{-\beta \varepsilon\left(I, m_{3}\right)}
$$

Thus the average polarization $\langle\bar{u}\rangle$ is obtained with $\mathcal{O}=\bar{u}$ and the probability distribution of polarizations $P(u)$ is obtained using $\mathcal{O}=\delta(u-\bar{u})$.

\section{Scaling and numerical evaluation}

To evaluate the integrals in eq. (3.33) for polarization averages, we need to know $\bar{u}$ as a function of the variables $\left(I, m_{3}, m_{z}, h\right)$. However, we calculate $\bar{u}$ using eq. (2.21), which provides it as a function of $e$ instead of $I$. One thus needs to calculate $e$ as a function of these variables and eliminate $e$ as an independent variable. This task is considerably simplfied by using scaling properties of the various quantities as a function of $I$. We write all quantities in term of the scaled variables,

$$
m_{z}^{*}=\frac{m_{z}}{I}, \quad m_{3}^{*}=\frac{m_{3}}{I}, \quad h^{*}=\frac{h}{I}, \quad e^{*}=\frac{e}{I}, \quad J_{\theta}^{*}=\frac{J_{\theta}}{I}
$$

Eq. (3.24) then becomes an equation for $J_{\theta}^{*}$ that is independent of $I$. We find $J_{\theta}^{*}$ as a function of $e^{*}$ and numerically invert the relationship to find $e^{*}\left(J_{\theta}^{*}, m_{z}^{*}, m_{3}^{*}, h^{*}\right)$. In fact, we only need $e^{*}$ for the adiabatically evolved state, i.e. for $J_{\theta}^{*}=\min (1-$ $\left.\left|m_{3}^{*}\right|, 1-\left|m_{z}^{*}\right|\right)$. With this value of $e^{*}$, we calculate $\bar{u}\left(m_{z}^{*}, m_{3}^{*}, h^{*}\right)$ from eq. (2.21). We may now express the ensemble average of $\bar{u}$ in the form

$$
\langle\bar{u}\rangle_{\mathrm{ar}}(x)=\sqrt{\frac{1-\alpha}{4 \pi}} \int_{0}^{\infty} \mathrm{d} \eta \sqrt{\eta} \int_{-1}^{1} \mathrm{~d} m_{\mathrm{z}}^{*} \int_{-1}^{1} \mathrm{~d} m_{3}^{*} \bar{u}\left(m_{\mathrm{z}}^{*} m_{3}^{*} x \sqrt{\eta}\right)
$$

where $x=\frac{\mu_{0} B}{k_{\mathrm{B}} T}$ is the dimensionless parameter in the Langevin function,

$$
\langle\bar{u}\rangle_{\mathrm{L}}=\operatorname{coth}\left(\frac{\mu_{0} B}{k_{\mathrm{B}} T}\right)-\frac{k_{\mathrm{B}} T}{\mu_{0} B}
$$

For the adiabatic rotor ensemble, there is an additional dimensionless parameter, the ratio of moments of inertia, which we write as

$$
\alpha=1-\mathcal{J}_{1} / \mathcal{J}_{3}
$$

It is clear on dimensional grounds that the polarization distribution $P(u)$ and the polarization $\bar{u}$ can be written as a function of $x$ and $\alpha$ alone. 
We now compare the average polarization with the Langevin function. We expect that $\langle\bar{u}\rangle_{a r}<\langle\bar{u}\rangle_{L}$, because of the presence of constraints in the adiabatic rotor ensemble that are absent in the full canonical ensemble associated with the Langevin funcion. The numerical evaluation of $\bar{u}$ using eq. (2.23) and (3.33) is shown in Fig. 1, for $\alpha=0$. For small value of $x$, i.e., in the limit of a weak field or high temperature, the Langevin function behaves like $\langle\bar{u}\rangle \simeq \frac{x}{3}$. This limit can also be extracted analytically for the adiabatic rotor ensemble. Here the derivative of the magnetization with respect to $h^{*}$ is given by

$$
\frac{\partial \bar{u}}{\partial h^{*}}=\frac{1}{4}\left(-1+3\left(m_{3}^{* 2}+m_{\mathrm{z}}^{* 2}\right)-5 m_{3}^{* 2} m_{\mathrm{z}}^{* 2}\right) \text {, }
$$

and hence

$$
\langle\bar{u}\rangle_{\mathrm{ar}} \simeq \frac{2}{9} x
$$

This result agrees with the quantum study, ref. ().

It is also interesting to compare the magnetization for the strong field or low temperature limit. Here the Langevin formula has an $x$-dependence as $\langle u\rangle_{\mathrm{L}} \simeq$ $1-1 / x$. To derive the corresponding limit for the adiabatic rotor ensemble, we first note that the polynomial $f(u)$ can be approximated by a quadratic function. We may then easily evaluate $J_{\theta}$ and $e^{*}$ as function of $m_{z}^{*}, m_{3}^{*}$ and $h^{*}$. The approximate magnetization for the state $\left(m_{\mathrm{x}}^{*} m_{3}^{*} h^{*}\right)$ is found to be

$$
\bar{u}=\frac{h^{*}-e^{*}}{2 h^{*}}=1-\sqrt{\frac{2}{h^{*}}}\left(1-\frac{\left|m_{\mathrm{z}}^{*}+m_{3}^{*}\right|}{2}\right) .
$$

Then the asymptotic form of the average polarization in the strong field limit is given by

$$
\langle\bar{u}\rangle_{\mathrm{ar}}=1-\frac{4}{3} \sqrt{\frac{2}{\pi x}} \text {, large } x .
$$

where the coefficient is $\frac{4}{3} \sqrt{\frac{2}{\pi}} \approx 1.06$. In all cases the magnetization for the adiabatic rotor ensemble is less than that of the canonical ensemble.

The deflection profile brings about much more rich information on the dynamics of magnetization of individual state of clusters. The calculated deflection profile through the Stern-Gerlach magnet is obtained calculating the ensemble average of the operator $\delta(u-\bar{u})$. In zero magnetic field and for $\alpha=0$, the profile function is given by

$$
P(u)=\int_{-1}^{1} \mathrm{~d} m_{\mathrm{z}}^{*} \int_{-1}^{1} \mathrm{~d} m_{3}^{*} \delta\left(u-m_{\mathrm{z}}^{*} m_{3}^{*}\right)=-2 \ln |u|,
$$

This function has a singularity at $u=0$, which becomes a peak of finite width as the magnetic field is increased. Fig. 2 shows the deflection profiles for several values of the Langevin parameter. For weak fields and moderate fields, the peak near $u=0$ is still recognizable. Besides the narrow peak, there is shoulder in the region of aligned polarizations which grows larger as the field gets stronger. This behavior is qualitatively similar to the observed deflection of $\mathrm{Gd}_{21}$ reported in ref. [3]. 


\section{Conclusion and discussions}

The magnetization in the adiabatic rotor ensemble is seen to behave quite smoothly as a function of the Langevin parameter $x$. The average polarization has the same order of magnitude as the Langevin function, but is always somewhat lower. Many experimental studies have used the Langevin function to interpret the deflection data, extracting a magnetic moment of the cluster. Our work points out that one needs to be careful that the system is actually the appropriate ensemble to make this analysis.

Rare earth clusters are likely to follow the adiabatic rotor ensemble at low temperatures, and the structure in the deflection profile would provide a clear footprint of the ensemble. In particular, the strong peak at $\bar{u}=0$ in weak fields, and the remainder of that peak in moderate fields are characteristics of the ensemble.

There is a body of data indicating nonmonotonic behavior of magnetization with magnetic field. This demands more intricate dynamics in the coupling of the spin to other degrees of freedom. Now we are working on an intermediate coupling model of electron spin with the rigid rotor.

\section{Acknowledgement}

This work was initiated at the Institute for Nuclear Theory and supported by their Grant FG06-90ER-40561. We also acknowledge discussions with W. deHeer and B. Mottelson.

\section{References}

1. D.M. Cox et al., Phys. Rev. B 32, 7290 (1985)

2. W. de Heer, P. Milani, and A. Chatelain, Phys. Rev. Lett. 65, 488 (1990)

3. J.P. Bucher, D.C. Douglass, and L.A. Bloomfield Phys. Rev. Lett. 66, 3052 (1991)

4. D.C. Douglass, J.P. Bucher, and L.A. Bloomfield, Phys. Rev. Lett. 68, 1774 (1992); D.C. Douglass et al., Phys. Rev. B 4712974 (1993)

5. I. Billas, J. Becker, and W. de Heer, Z. Phys. D 26, 325 (1993)

6. P.J. Jensen and K. H. Bennemann, Z. Phys. D 26, 246 (1993)

7. G.F. Bertsch and K. Yabana, Phys. Rev. A 49, 1930 (1994)

8. A. Maiti and L.M. Falicov, Phys. Rev. B 48, 13596 (1993)

9. H. Goldstein Classical Mechanics Addison-Wesley (1950) 


\section{$7 \quad$ Figure Captions}

Fig. 1. Average magnetization as a function of the Langevin parameter $x=\mu B / k T$.

Fig. 2. Probability distribution of the magnetization, $P(u)$, for three values of the Langevin parameter $x$. 


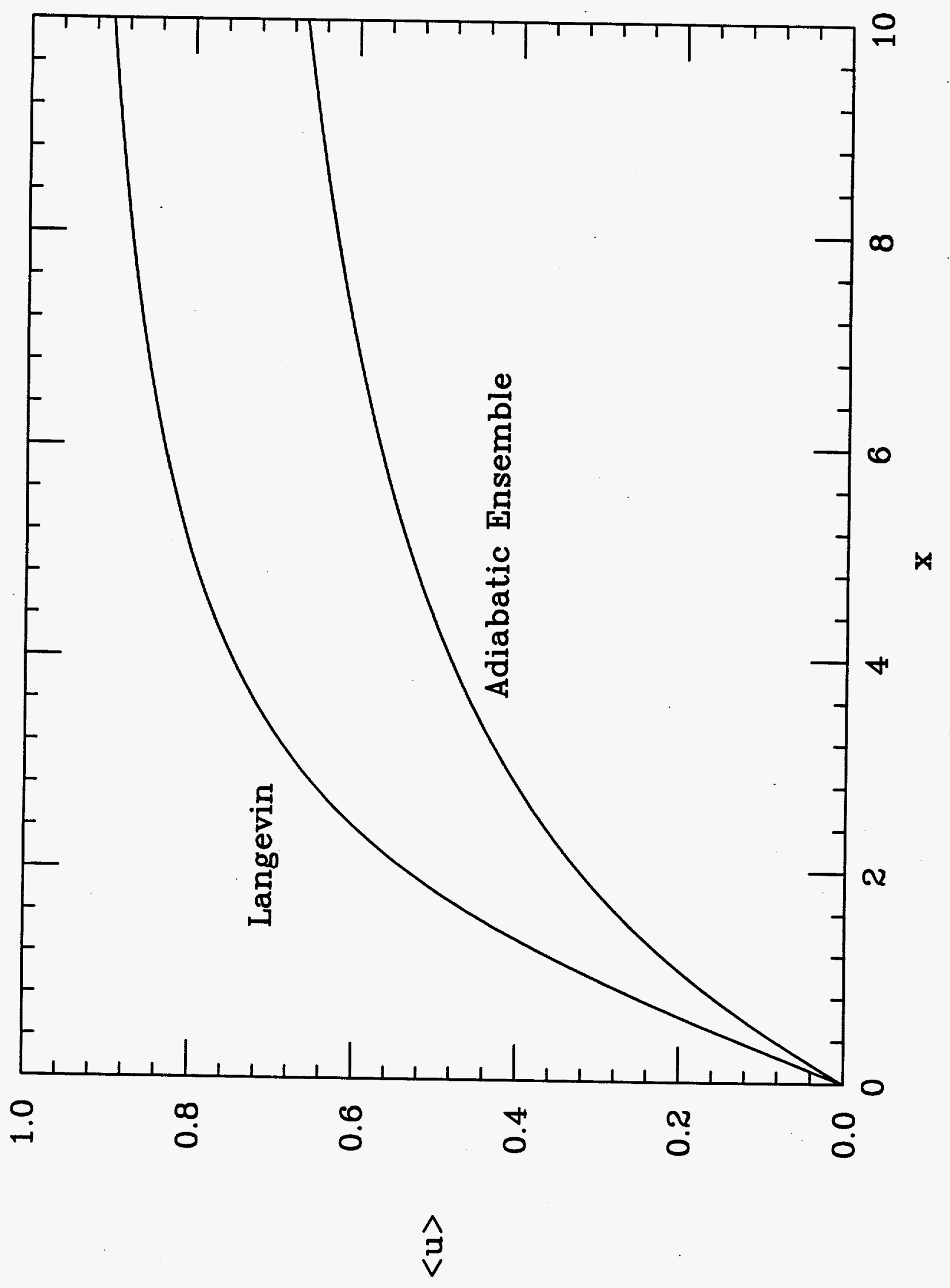




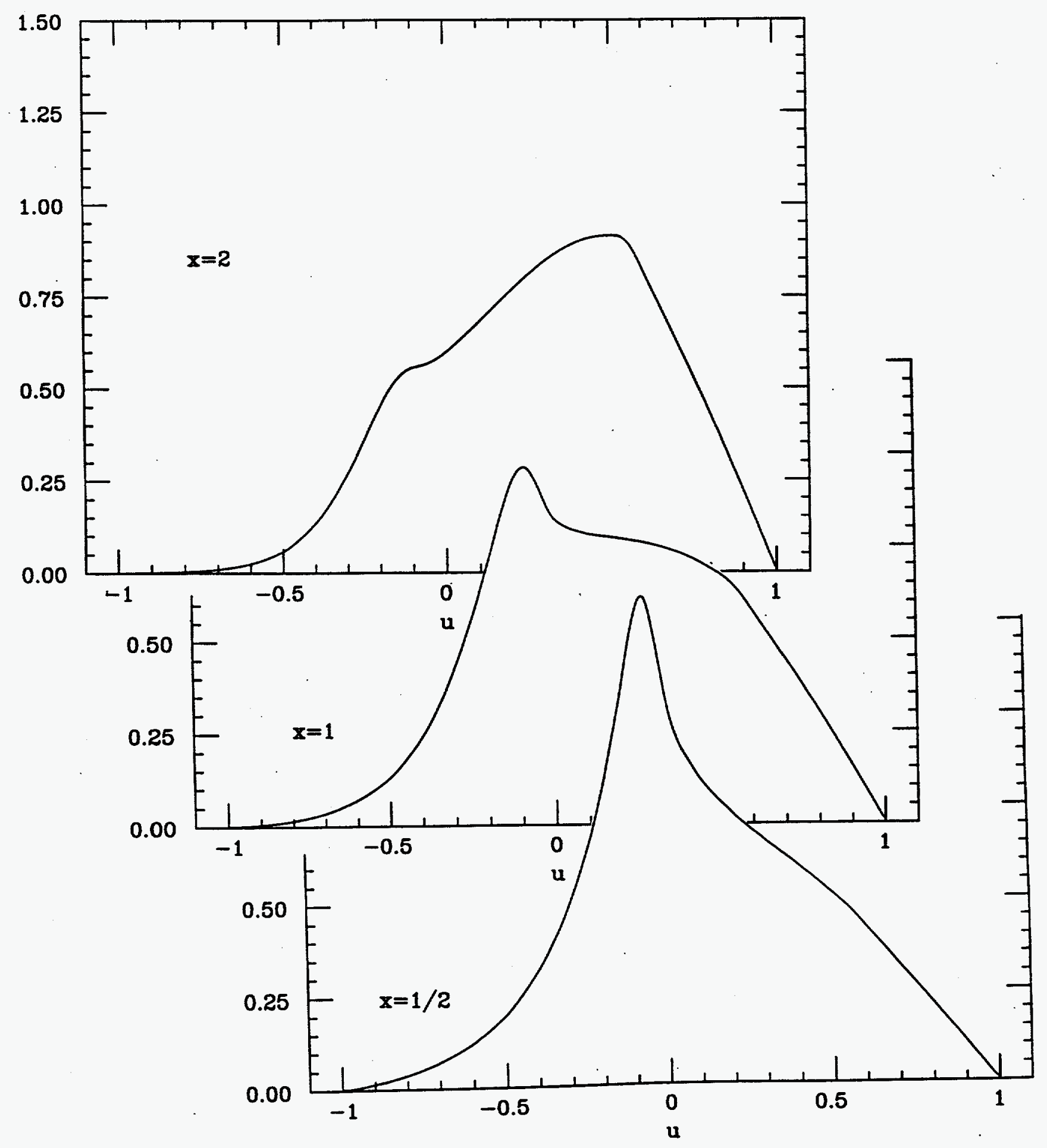

Fig. 2 